\title{
Long non-coding RNA BRAF-regulated IncRNA 1 promotes lymph node invasion, metastasis and proliferation, and predicts poor prognosis in breast cancer
}

\author{
JING JIANG, SHENG-HONG SHI, XU-JUN LI, LONG SUN, QI-DONG GE, CHAO LI and WEI ZHANG
}

Department of Breast Surgery, Ningbo No. 2 Hospital, Ningbo, Zhejiang 315000, P.R. China

Received May 20, 2017; Accepted February 2, 2018

DOI: $10.3892 / \mathrm{ol} .2018 .8513$

\begin{abstract}
Long non-coding RNAs (lncRNAs) are primary regulators of cancer development via their involvement in almost every aspect of cell biology. Recent studies have indicated that lncRNAs serve pivotal roles in breast cancer (BC) progression; however, to the best of our knowledge, the role of the lncRNA BRAF-regulated lncRNA 1 (BANCR) in $\mathrm{BC}$ has not yet been elucidated. The present study revealed that BANCR was overexpressed in BC cell lines and tissues, and could promote the clinical progression of disease, including increases in tumor size, lymph node metastasis and Tumor-Node-Metastasis stage. Furthermore, high BANCR expression was demonstrated to be associated with poor overall survival rates and early recurrence of $\mathrm{BC}$ in patients. Additionally, univariate and multivariate $\mathrm{COX}$ regression analyses identified high BANCR expression as an independent risk factor of poor prognosis of patients with BC. In addition, to verify the function of BANCR in BC cell lines, BANCR expression was silenced using short hairpin RNAs in MDA-MB-231 cells and overexpressed in MDA-MB-468 cells. An MTT assay and colony formation assay indicated that BANCR knockdown could suppress the proliferation of BC cells, whereas BANCR upregulation induced the proliferation of BC cells. Furthermore, BANCR silencing also reduced the migration and invasion of BC cells, as demonstrated via transwell migration and invasion assays. Consistently, the migration and invasion of BC cells increased upon BANCR ectopic overexpression in MDA-MB-468 cells. Mechanistically, matrix metallopeptidase 2/9 and epithelial-mesenchymal transition markers may be the potential targets of BANCR in regulating $\mathrm{BC}$ metastasis. In conclusion, BANCR overexpression could promote the clinical progression, metastasis and proliferation of $\mathrm{BC}$ and indicate poor prognosis of patients with BC.
\end{abstract}

Correspondence to: Professor Wei Zhang, Department of Breast Surgery, Ningbo No. 2 Hospital, 41 Xibei Street, Ningbo, Zhejiang 315000, P.R. China

E-mail: wzhangnb@163.com

Key words: long non-coding RNA BRAF-regulated lncRNA 1, metastasis, proliferation, prognosis, breast cancer
BANCR may therefore be a potential prognostic marker and therapeutic target of patients with BC.

\section{Introduction}

Recent data have indicated that breast cancer (BC) is the most common cancer in females in China in 2015 (1). In the last 2 decades, numerous advances have occurred in $\mathrm{BC}$ diagnosis and treatment; however, $\mathrm{BC}$ is a highly heterogeneous disease, and can exhibit different responses to existing treatments $(2,3)$. Thus, the molecular characteristics of $\mathrm{BC}$ and the progressive mechanisms underlying it require further investigation, which may lead to the elucidation of novel, effective prognostic markers and therapeutic targets.

Long non-coding RNAs (lncRNAs) are non-protein-coding transcripts of $>200 \mathrm{nt}$ (4). Evidence indicates that lncRNAs are essential participants in the regulation of protein-coding genes, maintenance of genomic integrity, dosage compensation, genomic imprinting, mRNA processing, and cell differentiation and development (5). The aberrant expression of lncRNAs is associated with multiple diseases, including cancer, immune diseases and neurological disorders (6-10). A number of lncRNAs, including metastasis associated lung adenocarcinoma transcript 1 (MALAT1), HOX transcript antisense RNA (HOTAIR) and H19, have been revealed to promote the development of BC (11-14), and a number of lncRNAs have been demonstrated to be potential prognostic markers and therapeutic targets of BC (13-18).

BRAF-regulated lncRNA 1 (BANCR) was identified by Flockhart et al (19) in 2012. Activating mutations in the BRAF oncogene are present in $>70 \%$ of melanomas, $90 \%$ of which produce the active mutant $\mathrm{BRAF}^{\mathrm{V} 600 \mathrm{E}}$ protein. BANCR was identified as one of the potentially novel intergenic transcripts on massively parallel cDNA sequencing (RNA-seq) that genetically analyzed matched normal human melanocytes with and without BRAF ${ }^{\mathrm{V} 600 \mathrm{E}}$ expression. Further investigations determined that BANCR was a recurrently overexpressed, previously unannotated 693-bp transcript on chromosome 9 , with a potential functional role in melanoma cell migration (19). Additionally, BANCR silencing suppressed melanoma cell migration, which could be rescued by the addition of the chemokine $\mathrm{C}-\mathrm{X}-\mathrm{C}$ motif chemokine ligand 11 to the medium (20). Since its identification, BANCR has been demonstrated to promote the progression and be associated 
with the poor prognosis of patients with multiple types of cancer, including esophageal squamous cell carcinoma (21), retinoblastoma (22), lung carcinoma (23), osteosarcoma (24) and gastrointestinal cancer (25); however, the functional role of BANCR in BC has not yet been elucidated.

The present study aimed to verify the potential role of BANCR in BC. To do this, the expression level of BANCR in $\mathrm{BC}$ cell lines and clinical samples was detected, and the association between BANCR expression and BC clinicopathological characteristics was statistically analyzed. Additionally, the prognostic value of BANCR expression was investigated. Furthermore, BANCR was overexpressed or silenced in BC cell lines to assess its function in motility and proliferation. In addition, the mechanism underlying BANCR regulating BC metastasis was also evaluated.

\section{Materials and methods}

Cell lines and cell culture. All human BC cell lines used in the current study, including non-invasive $\mathrm{BC}$ cell lines (MDA-MB-468, MCF7 and HCC1569), invasive BC cell lines (BT549 and MDA-MB-231) and normal human breast epithelial cell line, MCF10A, were obtained from Institute of Biochemistry and Cell Biology of the Chinese Academy of Sciences (Shanghai, China). MDA-MB-468, MCF7 and MDA-MB-231 cells were cultured in Dulbecco's modified Eagle's medium (Gibco; Thermo Fisher Scientific, Inc., Waltham, MA, USA). HCC1569, BT549 and MCF10A cells were cultured in RPMI-1640 medium (Gibco; Thermo Fisher Scientific, Inc.). The media contained $10 \%$ fetal bovine serum (FBS; Gibco; Thermo Fisher Scientific, Inc.), $100 \mathrm{U} / \mathrm{ml}$ penicillin and $100 \mathrm{mg} / \mathrm{ml}$ streptomycin and incubated at $37^{\circ} \mathrm{C}$ in an atmosphere containing $5 \% \mathrm{CO}_{2}$.

BC cell (MDA-MB-468 and MDA-MB-231) transfection was performed with Lipofectamine ${ }^{\circledR} 2000$ (Invitrogen; Thermo Fisher Scientific, Inc.), according to the manufacturer instructions. The number of cells transfected was indicated where used. Short interfering RNA (shRNA) BANCR silencing vectors (BANCR-shRNA.1 and BANCR-shRNA.2), a non-targeting shRNA (NC), pcDNA3.1-Vector and pcDNA3.1-BANCR were purchased from Genewiz, Inc. (Jiangsu, China). The mass of the plasmids and the shRNAs was $2 \mu \mathrm{g}$. After $48 \mathrm{~h}$, the transfected cells were subjected to subsequent experimentations.

Patient samples. BC tissues and paired non-cancerous tissues of 216 patients treated in The Ningbo No. 2 Hospital (Ningbo, China) between May, 2010 and October, 2012 were included in the present study. The following patients were excluded in the present study: i) Patients who received anticancer treatments prior to surgical resection; ii) patients who had $\geq 2$ malignances; and iii) patients who were diagnosed with recurrent BC upon surgery. Final diagnosis was concluded based on pathological results. All breast samples were obtained immediately following surgical resection, then frozen in liquid nitrogen and stored in $-80^{\circ} \mathrm{C}$ freezers until RNA was extracted. The clinicopathological information of the patients is presented in Table I. Notably, no patients with Tumor-Node-Metastasis (TNM) (26) stage IV disease were included in the present study due to the patients with stage IV disease all receiving anticancer treatments prior to surgical resection. Follow-up
Table I. Association between BANCR expression and clinicopathological characteristics of $\mathrm{BC}$ patients.

\begin{tabular}{|c|c|c|c|c|}
\hline \multirow[b]{2}{*}{ Parameters } & \multirow[b]{2}{*}{ Patients, n } & \multicolumn{2}{|c|}{$\begin{array}{c}\text { BANCR } \\
\text { expression, } \mathrm{n}\end{array}$} & \multirow[b]{2}{*}{ P-value } \\
\hline & & Low & High & \\
\hline Total & 216 & 91 & 125 & \\
\hline Age, years & & & & 0.335 \\
\hline$<50$ & 108 & 49 & 59 & \\
\hline$\geq 50$ & 108 & 42 & 66 & \\
\hline Menopausal status & & & & 0.845 \\
\hline Pre & 99 & 41 & 58 & \\
\hline Post & 117 & 50 & 67 & \\
\hline Tumor size, $\mathrm{cm}$ & & & & 0.008 \\
\hline$<2$ & 71 & 39 & 32 & \\
\hline$\geq 2$ & 145 & 52 & 93 & \\
\hline Lymph node status & & & & $<0.001$ \\
\hline Negative & 136 & 74 & 62 & \\
\hline Positive & 80 & 17 & 63 & \\
\hline TNM stage & & & & $<0.001$ \\
\hline $\mathrm{I}+\mathrm{II}$ & 138 & 73 & 65 & \\
\hline III & 78 & 18 & 60 & \\
\hline ER status & & & & 0.089 \\
\hline Negative & 90 & 44 & 46 & \\
\hline Positive & 126 & 47 & 79 & \\
\hline PR status & & & & 0.273 \\
\hline Negative & 102 & 39 & 63 & \\
\hline Positive & 114 & 52 & 62 & \\
\hline HER2 status & & & & 0.051 \\
\hline Negative & 121 & 58 & 63 & \\
\hline Positive & 95 & 33 & 62 & \\
\hline
\end{tabular}

BANCR, BRAF-regulated lncRNA 1; TNM, Tumor-Node-Metastasis; ER, estrogen receptor; PR, progesterone receptor; HER2, human epidermal growth factor receptor 2 .

studies were performed in the outpatient clinic in the Ningbo No. 2 Hospital (Ningbo, China), where physical examinations, laboratory analysis and computed tomography were performed if required. The deadline of follow-up studies was December, 2016. Overall survival (OS) time was defined as the time from the date of surgery to mortality or the latest date when censored. Recurrence-free survival (RFS) time was defined as the interval between the date of surgery and recurrence. The present study was approved by the Ethics Committee of the Ningbo No. 2 Hospital. Written informed consent was obtained from each patient, and all specimens were handled according to accepted ethical standards.

Total RNA extraction and revere transcription-quantitative polymerase chain reaction $(R T-q P C R)$ assay. Total RNA was extracted from clinical tissues or BC cell lines using TRIzol ${ }^{\circledR}$ reagent (Invitrogen; Thermo Fisher Scientific, Inc.), according 
to manufacturer's protocol. First-strand cDNA was generated with the extracted total RNA using the Reverse Transcription System kit (Invitrogen; Thermo Fisher Scientific, Inc.). The expression level of the target gene was evaluated by RT-qPCR with the standard SYBR ${ }^{\circledR}$-Green PCR kit (Invitrogen; Thermo Fisher Scientific, Inc.) on the ABI 7500 system (Applied Biosystems; Thermo Fisher Scientific, Inc.). The thermocycling conditions were as follows: initial denaturation at $95^{\circ} \mathrm{C}$ for $5 \mathrm{~min}, 40$ cycles of denaturation at $95^{\circ} \mathrm{C}$ for $30 \mathrm{sec}$, annealing at $50^{\circ} \mathrm{C}$ for $30 \mathrm{sec}$ and extension at $72^{\circ} \mathrm{C}$ for $30 \mathrm{sec}$. Melting curve analysis was used to monitor the specificity of the PCR products and the $2^{-\Delta \Delta \mathrm{Cq}}$ method (27) was utilized to evaluate the relative expression level of the target gene. All experiments were performed in triplicate with GAPDH serving as the internal control. The primers used in the present study were: BANCER forward, 5'-ACAGGACTCCATGGCAAACG-3' and reverse, 5'-ATGAAGAAAGCCTGGTGCAGT-3'; matrix metallopeptidase 2 (MMP2) forward, 5'-AAGGATGGCAAG TACGGCTT-3' and reverse, 5'-CGCTGGTACAGCTCTCAT ACTT-3'; MMP9 forward, 5'-ACCTCGAACTTTGACAGC GAC-3' and reverse, 5'-GAGGAATGATCTAAGCCCAGC-3'; MMP14 forward, 5'-CGATGTGGTGTTCCAGACAA-3' and reverse, 5'-TGGATGCAGAAAGTGATTTC-3'; epithelial cadherin (E-cadherin) forward, 5'-CATTGCCACACATAC ACTCTCTTCT-3' and reverse, 5'-CGGTTACCGTGATCA AAATCTC-3'; Vimentin forward, 5'-GGAACAGCATGTCCA AATCG-3' and reverse, 5'-GCACCTGTCTCCGGTACTCA-3'; GAPDH forward, 5'-GCACCGTCAAGGCTGAGAAC-3' and reverse, 5'-GGATCTCGCTCCTGGAAGATG-3'.

Cell proliferation assays. Cell proliferation ability was assessed with MTT and colony-formation assays. In the MTT assay, transfected BC cells and corresponding control cells were seeded in 96-well plates. MTT (Sigma-Aldrich; Merck KGaA, Darmstadt, Germany) reagent was added at $0,24,48,72$ and $96 \mathrm{~h}$, followed by incubation of the plates at $37^{\circ} \mathrm{C}$ for another $2 \mathrm{~h}$. Subsequently, dimethyl sulfoxide was used to solubilize the crystals. Finally, the absorbance was measured at $450 \mathrm{~nm}$ using a Multiskan ${ }^{\circledR}$ Spectrum system (Thermo Fisher Scientific, Inc.).

For the colony formation assay, transfected cells $\left(1 \times 10^{3}\right.$ cells per dish) were seeded into a 6 -well plate and incubated at $37^{\circ} \mathrm{C}$, and the medium containing $10 \%$ FBS was replaced every 3 days. After 2 weeks, formed colonies were fixed with pure methanol for $20 \mathrm{~min}$ and stained with $0.1 \%$ crystal violet for $20 \mathrm{~min}$ at room temperature. Images of the visible colonies were captured with a light microscope (x10, magnification) and counted manually.

Cell migration and invasion assays. For the migration assay, cells $\left(2 \times 10^{4}\right)$ were transfected $24 \mathrm{~h}$ prior to being suspended in serum-free medium and then seeded into the upper side of the Transwell chamber ( $8 \mu \mathrm{m}$ pore size) (BD Biosciences, Franklin Lakes, NJ, USA). The lower side of the chamber was filled with medium containing $10 \%$ FBS, serving as a chemoattractant. After incubation for $24 \mathrm{~h}$, cells invaded through the membrane were fixed in $4 \%$ paraformaldehyde for $30 \mathrm{~min}$ and stained with $0.1 \%$ crystal violet for another $30 \mathrm{~min}$ at room temperature. For quantitative analysis, images of cells adhered to the lower surface were captured with a light microscope (x10) and three random fields were counted. For the invasion assay, Matrigel (BD Biosciences) was pre-coated in the upper chamber of the Transwell chamber and the assay was performed as it was in the migration assay. Three independent experiments were performed for each experiment.

Statistical analysis. Statistical analyses were performed with the SPSS 17.0 software (SPSS, Inc., Chicago, IL, USA). Data are presented as mean \pm standard error of the mean. Statistical comparisons of qualitative data were produced with the $\chi^{2}$ test. Quantitative data was compared by two-tailed Student's t-test or analysis of variance test followed by Dunnett's test. The Kaplan-Meier method was used to estimate survival, and the survival difference was compared using the log-rank test. Survival data were evaluated using univariate and multivariate Cox proportional hazards models, and variables with a value of $\mathrm{P}<0.05$ in univariate analysis were further analyzed in subsequent multivariate Cox regression analysis. $\mathrm{P}<0.05$ was considered to indicate a statistically significant difference.

\section{Results}

Significant BANCR overexpression in BC cell lines and tissues. The expression level of BANCR in BC cell lines was examined via RT-qPCR to investigated its functional role; the results of this analysis demonstrated that BANCR was upregulated in BC cell lines, compared with normal human breast epithelial cell line MCF10A (Fig. 1A). In addition, the BANCR expression level in invasive BC cell lines was significantly higher than that in the noninvasive BC cell lines (Fig. 1A). The expression of BANCR was also assessed in 216 $\mathrm{BC}$ and paired non-cancerous tissues, the results of which indicated that BANCR was significantly overexpressed in BC tissues, compared with paired non-cancerous tissues $(\mathrm{P}<0.01$; Fig. 1B). BANCR overexpression in BC cell lines and tissues indicated that it may serve an oncogenic role in $\mathrm{BC}$.

Association between BANCR overexpression and prognosis of patients with $B C$. The clinical significance of BANCR expression in BC was further identified by analyzing the association between BANCR expression and the clinicopathological characteristics of patients with $\mathrm{BC}$, who were dichotomized for statistical analysis. All of the patients were determined to exhibit either low or high BANCR expression, with the BANCR mean expression level (1.9) serving as the cutoff value. The patients with BANCR level less than 1.9 were classified into the low BANCR expression group. Notably, BANCR overexpression was determined to be significantly associated with a larger tumor size $(\mathrm{P}=0.008)$, lymph node metastasis $(\mathrm{P}<0.001)$ and advanced TNM stage $(\mathrm{P}<0.001$; Table $\mathrm{I})$. The association between BANCR expression and the prognosis of patients with $\mathrm{BC}$ was calculated using the Kaplan-Meier method and compared with the log-rank test. As expected, patients with a high BANCR expression were demonstrated to have a poorer OS rate $(\mathrm{P}=0.006)$ and reduced $\mathrm{RFS}$ period $(\mathrm{P}=0.010)$, compared with patients exhibiting low BANCR. (Fig. 1C and D). Furthermore, univariate analysis indicated that positive lymph node status [hazard ratio $(\mathrm{HR})=2.140$, $95 \%$ confidence interval $(\mathrm{CI})=1.245-3.650 ; \mathrm{P}=0.005]$, advanced TNM stage $(\mathrm{HR}=1.623,95 \% \mathrm{CI}=1.115-2.361 ; \mathrm{P}=0.011)$ and high 
A

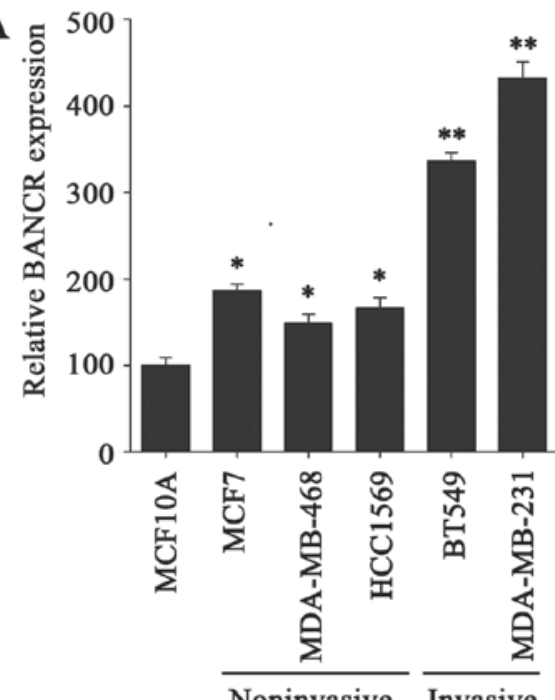

$\mathrm{C}$

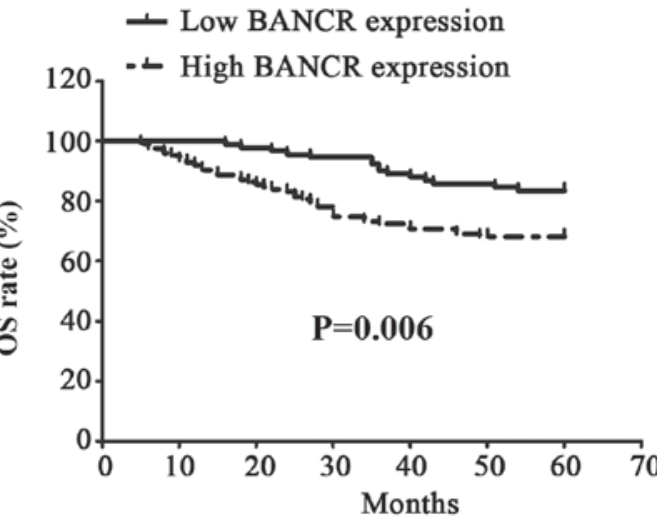

B

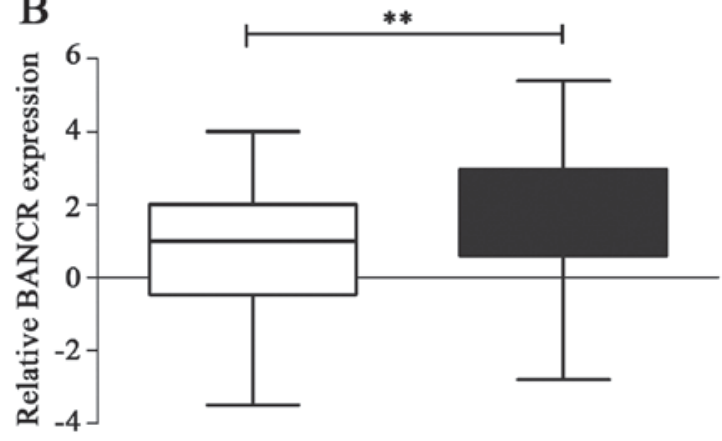

Non-cancerous tissues

Breast cancer tissues

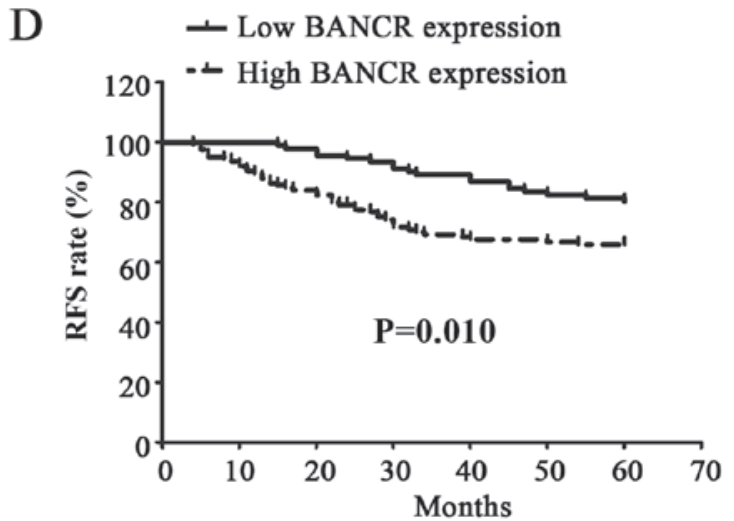

Figure 1. IncRNA BANCR expression is upregulated in BC cells and tissues, which is associated with poor prognosis of patients with BC. (A) The expression of BANCR was detected via RT-qPCR in normal human breast epithelial cell line, MCF10A, noninvasive BC cell lines (MDA-MB-468, MCF7 and HCC1569) and invasive BC cell lines (BT549 and MDA-MB-231). (B) The expression of BANCR in non-cancerous tissues and BC tissues was verified by RT-qPCR. (C) The Kaplan-Meier method and log-rank test were utilized to analyze the difference in OS rate between the low- and high-BANCR-expression groups. (D) The Kaplan-Meier method and log-rank test were used to analyze the difference in RFS rate between the low- and high-BANCR-expression groups. Statistical significance was determined using Student's t-test between indicated columns in (A and B), and by log-rank test in (C and D). "P<0.05, ${ }^{* *} \mathrm{P}<0.01$. BC, breast cancer; RT-qPCR, reverse transcription-quantitative polymerase chain reaction; BANCR, BRAF-regulated lncRNA 1; OS, overall survival; RFS, recurrence-free survival.

BANCR expression $(\mathrm{HR}=1.614,95 \% \mathrm{CI}=1.353-1.989 ; \mathrm{P}<0.001)$ were three risk factors for patients with $\mathrm{BC}$ with a poor OS rate. Additionally, the same three factors were also identified as risk factors of a reduced RFS, positive lymph node status $(\mathrm{HR}=1.999,95 \% \mathrm{CI}=1.205-3.317 ; \mathrm{P}=0.007)$, advanced TNM stage $(\mathrm{HR}=1.471,95 \% \mathrm{CI}=1.038-2.085 ; \mathrm{P}=0.030)$ and high BANCR expression $(\mathrm{HR}=1.575,95 \% \mathrm{CI}=1.317-1.883$; $\mathrm{P}<0.001$; Tables II and III). However, further analysis of these factors using multivariate analysis, only high BANCR expression was highlighted as an independent risk factor of patients with $\mathrm{BC}$ with a poor $\mathrm{OS}$ rate $(\mathrm{HR}=1.585,95 \% \mathrm{CI}=1.298-1.935 ; \mathrm{P}<0.001)$ and early recurrence $(\mathrm{HR}=1.532,95 \% \mathrm{CI}=1.272-1.844$; $\mathrm{P}<0.001$; Tables II and III). These data indicated that BANCR overexpression could promote the clinical progression of $\mathrm{BC}$ and predicts poor prognosis of patients with $\mathrm{BC}$.

BANCR promotes BC metastasis and proliferation. Next, the functional role of BANCR was detected in BC cell lines. RNA interference was performed in MDA-MB-231 cells, owing to the relatively high expression level of BANCR; BANCR ectopic overexpression was conducted in MDA-MB-468 cells, owing to the relatively low expression level of BANCR (Fig. 1A). The expression of BANCR in MDA-MB-231 and MDA-MB-468 cells was demonstrated to be notably downregulated or upregulated following transfection, respectively (Fig. 2A and B).

The role of BANCR on the proliferation of BC cells was detected using MTT and colony formation assays. The results of the MTT assay indicated that BANCR deficiency in MDA-MB-231 cells notably reduced its proliferation ability $(\mathrm{P}<0.05$; Fig. $2 \mathrm{C})$, while BANCR overexpression in MDA-MB-468 cells promoted the proliferation $(\mathrm{P}<0.05$; Fig. 2D). Similar results were obtained from the colony formation assay $(\mathrm{P}<0.05$; Fig. $2 \mathrm{E}$ and $\mathrm{F})$. Migration and invasion assays were performed to analyze the association between BANCR expression levels and the migration and invasion of BC cells. The results demonstrated that BANCR silencing could significantly inhibit the migration and invasion of MDA-MB-231 cells ( $\mathrm{P}<0.05$; Fig. $3 \mathrm{~A}$ and B). Contrastingly, BANCR overexpression significantly facilitated the migration and invasion of MDA-MB-468 cells ( $\mathrm{P}<0.05$; Fig. $3 \mathrm{C}$ and $\mathrm{D})$. Notably, as shown in Fig. 3A and B, statistical analysis revealed 
Table II. Univariate and multivariate Cox regression analysis of overall survival of BC patients.

\begin{tabular}{lcccc}
\hline & \multicolumn{2}{c}{ Univariate analysis } & & Multivariate analysis \\
\cline { 2 - 3 } Parameters & HR $(95 \% \mathrm{CI})$ & P-value & HR (95\% CI) \\
\hline Age $(\geq 50$ vs. $<50$ years) & $1.031(0.604-1.760)$ & 0.911 & \\
Menopausal status (pre vs. post) & $1.067(0.625-1.822)$ & 0.812 & \\
Tumor size ( $\geq 2$ vs. $<2$ cm) & $1.117(0.629-1.983)$ & 0.706 & \\
Lymph node status (negative vs. positive) & $2.140(1.254-3.650)$ & 0.005 & $1.332(0.639-2.780)$ \\
TNM (stage I+II vs. III) & $1.623(1.115-2.361)$ & 0.011 & $1.120(0.665-1.885)$ \\
ER status (negative vs. positive) & $0.993(0.577-1.708)$ & 0.979 & 0.444 \\
PR status (negative vs. positive) & $1.100(0.643-1.882)$ & 0.727 & & 0.671 \\
HER2 status (negative vs. positive) & $1.265(0.742-2.157)$ & 0.387 & \\
BANCR expression (high vs. low) & $1.614(1.353-1.989)$ & $<0.001$ & $1.585(1.298-1.935)$ \\
\hline
\end{tabular}

HR, hazard ratio; CI, confidence interval; BANCR, BRAF-regulated lncRNA 1; TNM, Tumor-Node-Metastasis; ER, estrogen receptor; PR, progesterone receptor; HER2, human epidermal growth factor receptor 2.

Table III. Univariate and multivariate Cox regression analysis of recurrence-free survival of patients with breast cancer.

\begin{tabular}{|c|c|c|c|c|}
\hline \multirow[b]{2}{*}{ Parameters } & \multicolumn{2}{|c|}{ Univariate analysis } & \multicolumn{2}{|c|}{ Multivariate analysis } \\
\hline & HR $(95 \% \mathrm{CI})$ & P-value & HR $(95 \% \mathrm{CI})$ & P-value \\
\hline Age $(\geq 50$ vs. $<50$ years $)$ & $0.979(0.589-1.629)$ & 0.936 & & \\
\hline Menopausal status (pre vs. post) & $1.047(0.631-1.739)$ & 0.859 & & \\
\hline Tumor size ( $\geq 2$ vs. $<2 \mathrm{~cm}$ ) & $1.011(0.591-1.729)$ & 0.968 & & \\
\hline Lymph node status (negative vs. positive) & $1.999(1.205-3.317)$ & 0.007 & $1.421(0.703-2.872)$ & 0.328 \\
\hline TNM (stage I+II vs. III) & $1.471(1.038-2.085)$ & 0.030 & $0.992(0.703-2.872)$ & 0.328 \\
\hline ER status (negative vs. positive) & $1.024(0.611-1.717)$ & 0.927 & & \\
\hline PR status (negative vs. positive) & $0.997(0.600-1.655)$ & 0.989 & & \\
\hline HER2 status (negative vs. positive) & $1.268(0.764-2.103)$ & 0.358 & & \\
\hline BANCR (high vs. low) & $1.575(1.317-1.883)$ & $<0.001$ & $1.532(1.272-1.844)$ & $<0.001$ \\
\hline
\end{tabular}

HR, hazard ratio; CI, confidence interval; BANCR, BRAF-regulated lncRNA 1; TNM, Tumor-Node-Metastasis; ER, estrogen receptor; PR, progesterone receptor; HER2, human epidermal growth factor receptor 2.

that BANCR interference left $\sim 50 \%$ of invasive cells able to permeate the membrane compared with the NC group, which may have resulted from the involvement of a wide range of molecules in cell biology, including protein-coding genes and microRNAs. IncRNAs function to regulate cell biology. Taken together, these data indicate that BANCR could promote the proliferation and metastasis of $\mathrm{BC}$ cells.

BANCR promotes BC metastasis through regulating MMP2/9 and epithelial-mesenchymal transition (EMT). To investigate the mechanisms underlying the BANCR-dependent promotion of BC metastasis, the expression of invasive markers, including MMP2, MMP9 and MMP14, were quantified by RT-qPCR (Fig. 4). Expression of MMP2/9 were significantly suppressed in BANCR-knockdown MDA-MB-231 cells, whereas MDA-MB-468 cells overexpressing BANCR exhibited significantly higher MMP2/9 expression (Fig. 4A, B, F and G). The expression level of MMP14 was relatively stable despite the overexpression or knockdown of BANCR (Fig. 4C and H).
EMT is a fundamental process in cancer cell metastasis (28). The expression of the epithelial marker E-cadherin and the mesenchymal marker vimentin was thus analyzed using RT-qPCR. BANCR upregulation increased the expression of vimentin and reduced the expression of E-cadherin. Contrastingly, the vimentin expression level was decreased and E-cadherin expression level was upregulated following BANCR silencing (Fig. 4D, E, I and J).

\section{Discussion}

lncRNAs are regarded as pivotal regulators in the development and progression of cancer $(8,29)$. A number of lncRNAs have been revealed as pivotal regulators and associated with clinical outcomes in patients with $\mathrm{BC}$ through various regulating pathways (17,30). A number of lncRNAs, including HOTAIR, lncRNA activated by TGF- $\beta$, breast cancer anti-estrogen resistance 4 , urothelial cancer-associated 1 and growth arrest specific 5 , have been demonstrated to be associated with 

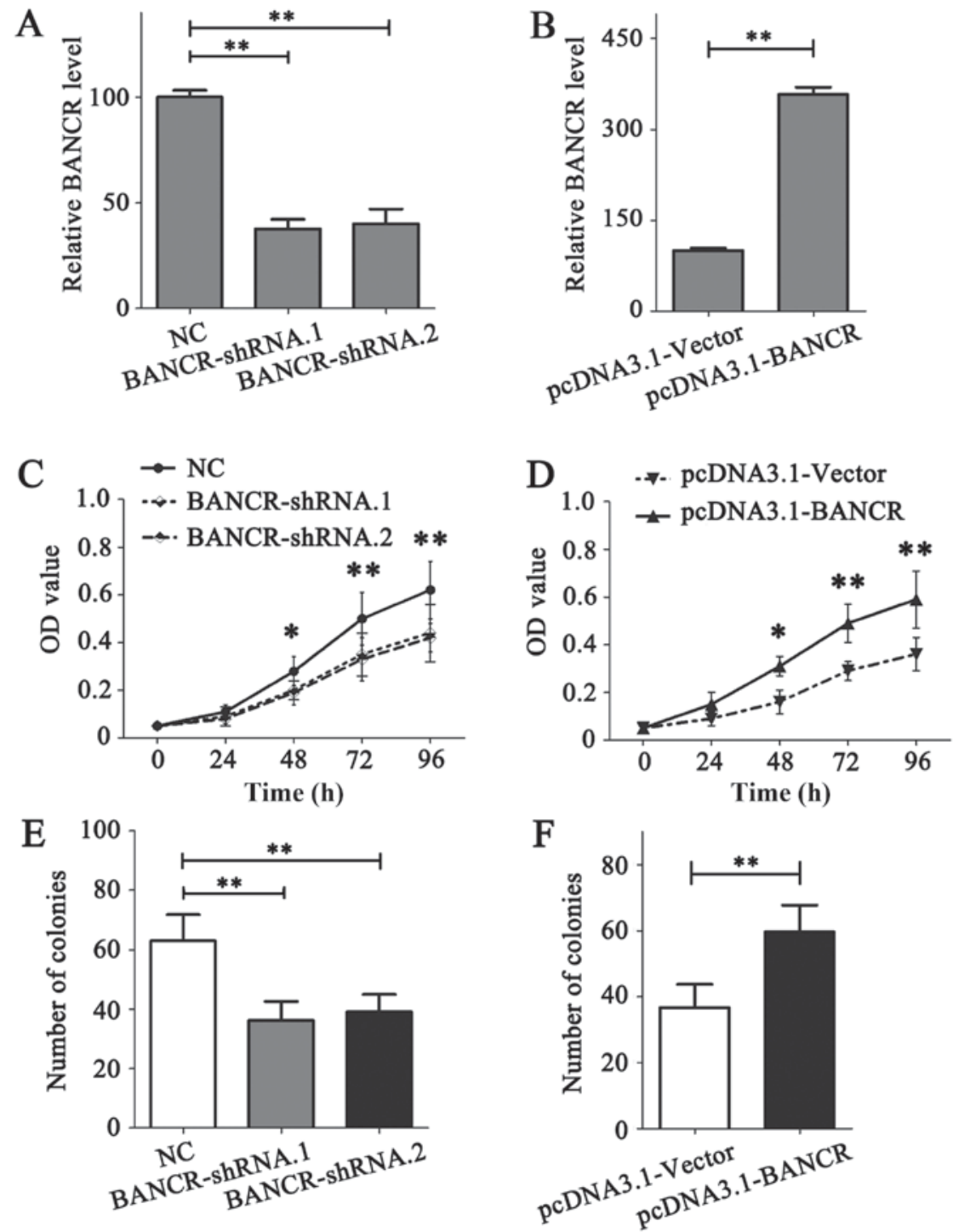

Figure 2. Upregulation of BANCR could promote the proliferation of breast cancer cells. (A) The expression of BANCR in MDA-MD-231 cells was detected via RT-qPCR following transfection. (B) The expression of BANCR in MDA-MD-468 cells was detected via RT-qPCR following transfection. (C) An MTT assay was conducted to assess the role of BANCR on the proliferation of MDA-MB-231 cells. (D) An MTT assay was conducted to detect the role of BANCR on the proliferation of MDA-MB-468 cells. (E) A colony formation assay was performed to detect the role of BANCR on the proliferation of MDA-MB-231 cells. (F) A colony-formation assay was performed to detect the role of BANCR on the proliferation of MDA-MB-468 cells. Statistical significance was determined using Student's t-test between the indicated columns in (A-F). ${ }^{*} \mathrm{P}<0.05,{ }^{* *} \mathrm{P}<0.01$. RT-qPCR, reverse transcription-quantitative polymerase chain reaction; BNCR, BRAF-regulated lncRNA 1; OD, optical density; NC, negative control; shRNA, short hairpin RNA.

anticancer drug resistance (31). The lncRNAs 91H (32), IncRNA inhibiting metastasis (33) and eosinophil granule ontogeny transcript (16) have been indicated to promote an aggressive $\mathrm{BC}$ phenotype. BC is a highly heterogeneous disease, and lncRNAs also display specific expression patterns in different subtypes of BC $(11,34)$. For example, HOTAIR only provided a prognostic insight in patients with ER-negative BC (34), whereas MALAT1 overexpression was associated with poor RFS in tamoxifen treated patients with ER-positive BC, and may serve as a potential biomarker to predict endocrine treatment sensitivity (11). Each patient's lncRNA expression signature may provide a novel method for individualized anticancer treatments. Several lncRNAs have already been highlighted as prognostic markers and therapeutic targets in BC (30). However, the significance of BANCR has not yet been identified.

Upregulation of BANCR has been observed in multiple cancer types, including gastrointestinal cancer (25), lung cancer (35), esophageal squamous cell carcinoma (21), papillary thyroid carcinoma (36), endometrial cancer (37), osteosarcoma (24) and retinoblastoma (22). In demonstrating the functional role of BANCR in BC, the present study first observed that BANCR was overexpressed in BC cell lines and clinical tissues. It has been reported that BANCR overexpression accelerates the progression of various cancer types $(21,23,24,35-41)$. The present study determined that high BANCR expression was associated with a larger tumor size, lymph node metastasis and advanced TNM stages in patients with $\mathrm{BC}(\mathrm{P}<0.05)$. However, further research is required to investigate the association between BANCR expression and distant metastasis. Additionally, high BANCR expression predicted a poor $\mathrm{OS}$ rate $(\mathrm{HR}=1.585,95 \% \mathrm{CI}=1.298-1.935$; $\mathrm{P}<0.001)$ and early recurrence $(\mathrm{HR}=1.532,95 \% \mathrm{CI}=1.272-1.844$; $\mathrm{P}<0.001)$ in patients with $\mathrm{BC}$. BANCR upregulation was also identified as an independent risk factor of poor OS and RFS rates 

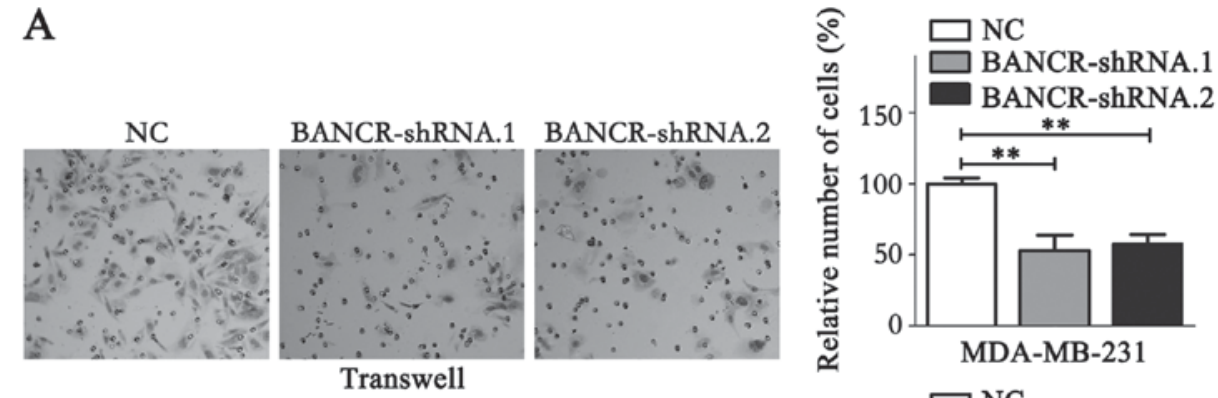

B

$\mathrm{NC}$

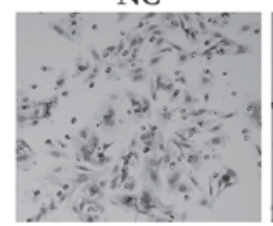

BANCR-shRNA.1

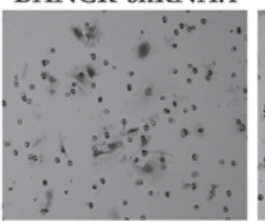

Matrigel

C

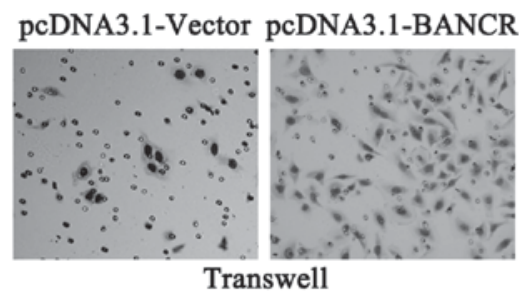

D

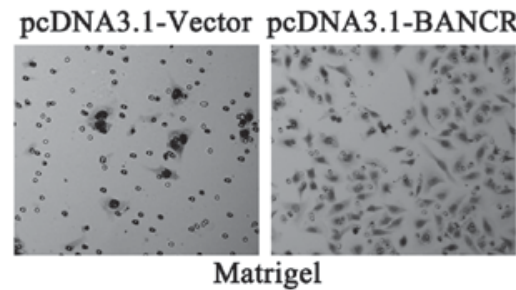

BANCR-shRNA.2

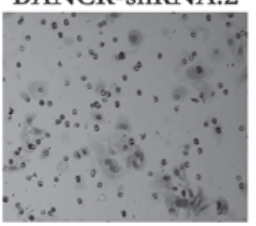

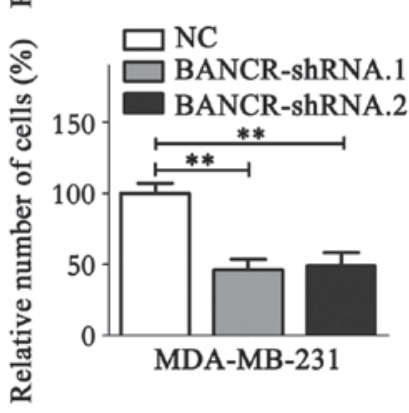
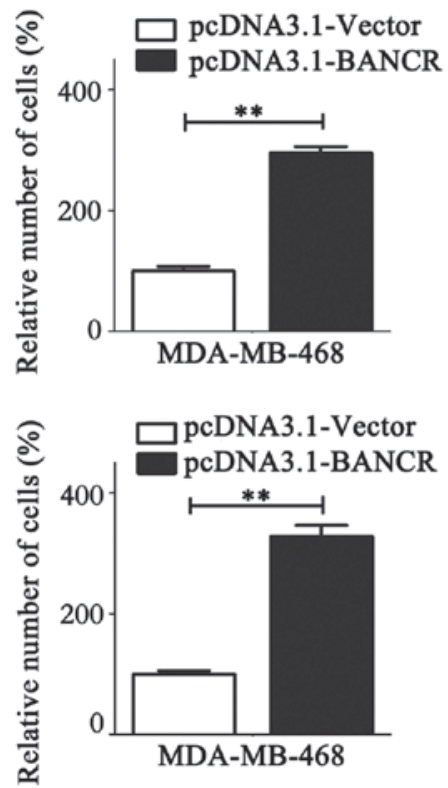

Figure 3. BANCR could promote the metastasis of breast cancer cells. (A) The migratory ability of MDA-MB-231 cells was monitored via a transwell migration assay following transfection. (B) The invasive ability of MDA-MB-231 cells was detected via a Matrigel invasion assay following transfection. (C) The migratory ability of MDA-MB-468 cells was monitored via a transwell migration assay following transfection. (D) The invasive ability of MDA-MB-468 cells was detected via a Matrigel invasion assay following transfection. Statistical significance was determined via Student's t-test between the indicated columns in (A-D). ${ }^{* *} \mathrm{P}<0.01$. NC, negative control; BNCR, BRAF-regulated lncRNA 1.

for patients with BC. In vitro assays demonstrated that BANCR could facilitate the migration, invasion and proliferation of BC cells. Mechanistically, the expression of invasive markers, MMP2 and MMP9 was positively associated with BANCR expression. BANCR was also demonstrated to accelerate EMT in BC cells. However, as a limitation of the present study, the aforementioned observations were only obtained in the in vitro setting, meaning that further research is required to verify the association between BANCR and markers of invasion in patient samples. To the best of our knowledge, the present study is the first to demonstrate the oncogenetic role of BANCR in BC and to indicate that BANCR could serve as an effective prognostic marker and therapeutic target in BC.

The detailed mechanisms by which BANCR regulates cancer biology have been investigated in a number of studies (37-39,41-43). BANCR actively functions as a regulator of EMT during non-small cell lung cancer and colorectal cancer metastasis $(42,43)$. The mitogen-activated protein kinase signaling pathway has been implicated as the target of BANCR in promoting the proliferation of melanoma and endometrial cancer $(37,38)$. Additionally, Zhang et al (39) revealed that BANCR could promote gastric cancer cell proliferation by regulating nuclear factor- $\kappa \mathrm{B} 1$, whereas $\mathrm{p} 21$ was indicated to be the target gene of BANCR, affecting the proliferation of colorectal cancer cells (41). Notably, the mechanisms underlying BANCR functioning vary in different cancer types, which requires further study.

In conclusion, the present study demonstrated that BANCR was overexpressed in BC, and this expression was significantly associated with the clinical progression of $\mathrm{BC}$. 

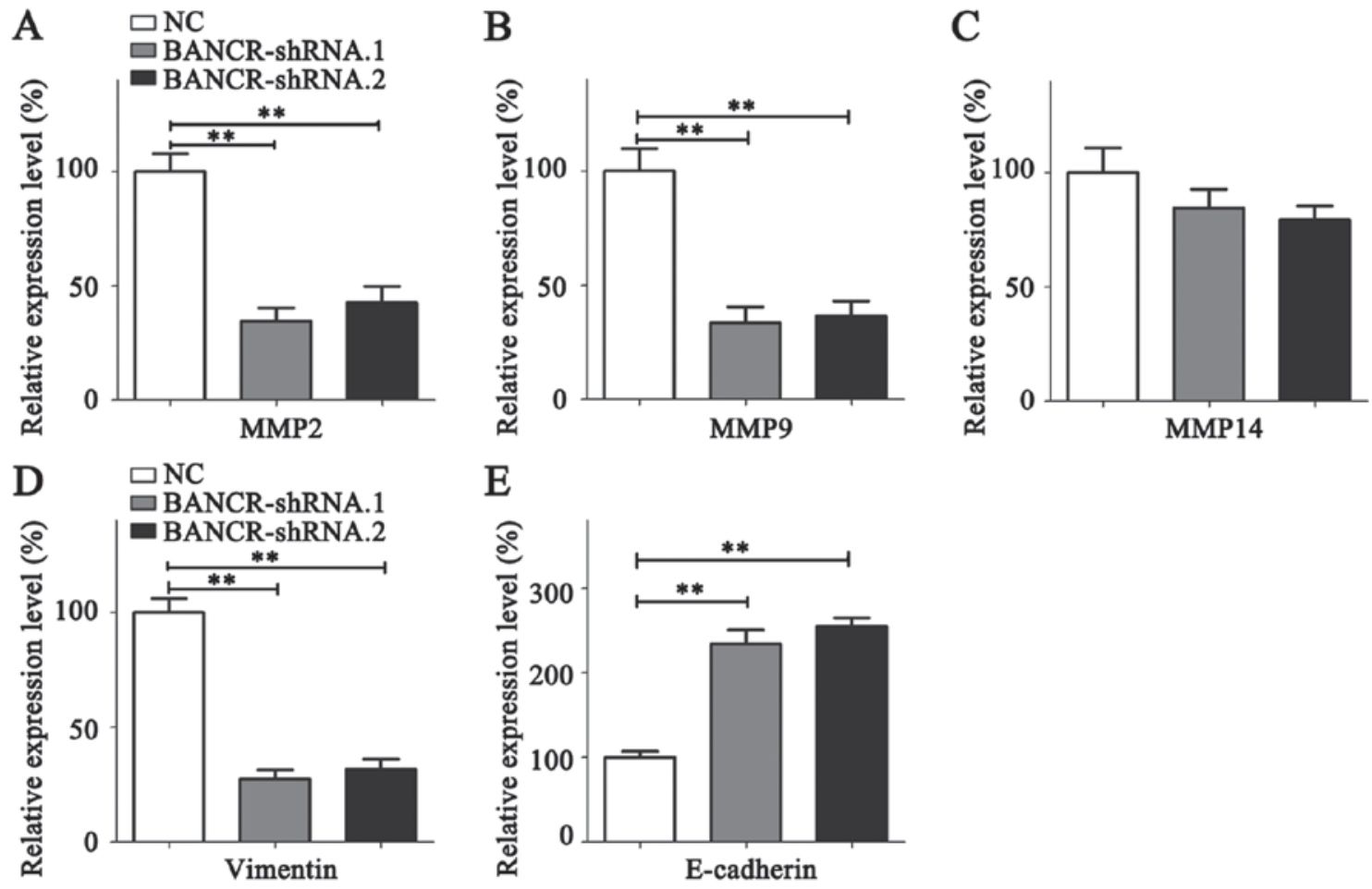

$\mathrm{E}$
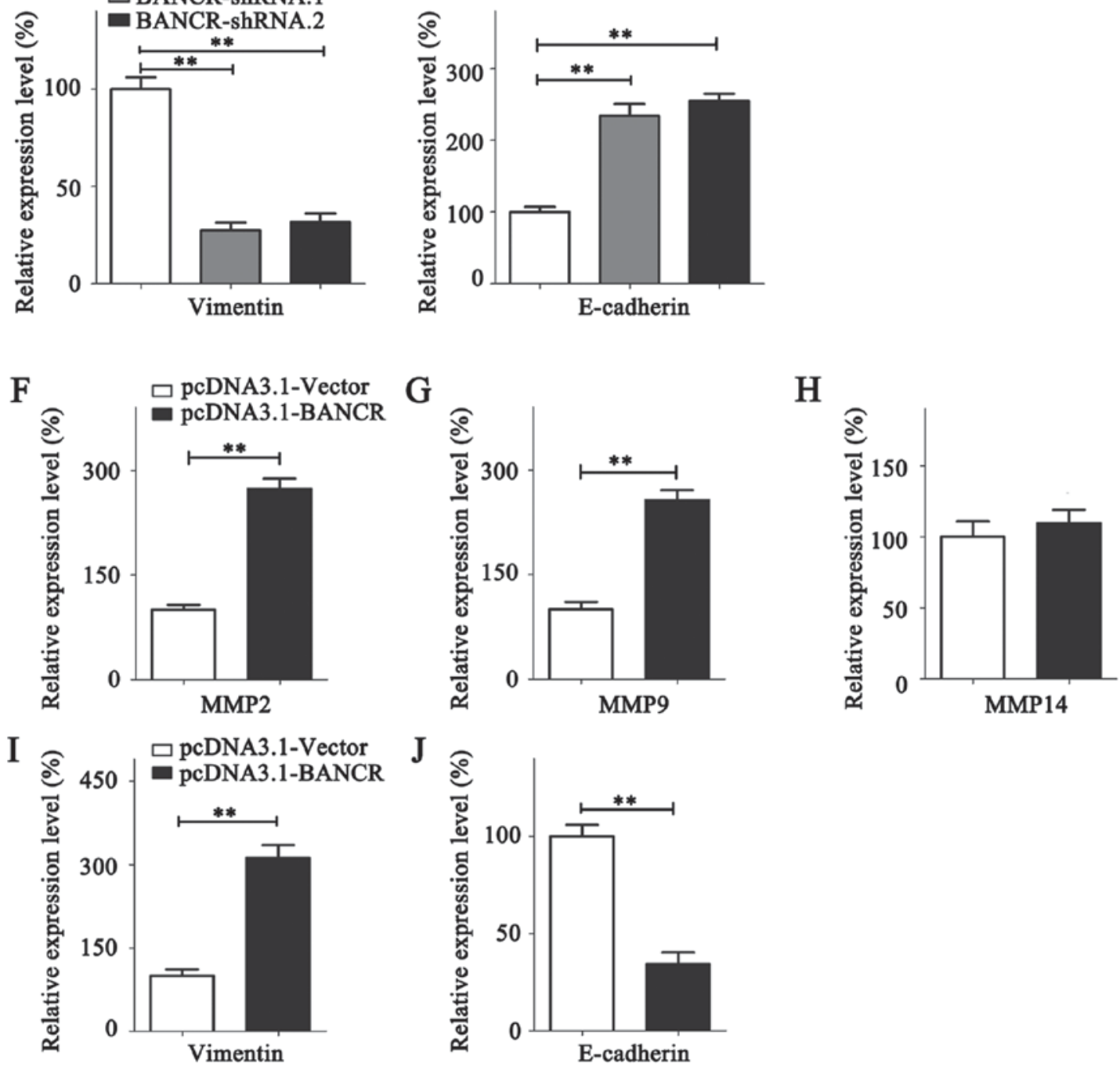

Figure 4. BANCR promotes the metastasis of breast cancer cells by regulating MMP2/9 and the epithelial-mesenchymal transition. The expression of (A) MMP2, (B) MMP9, (C) MMP14, (D) Vimentin and (E) E-cadherin was analyzed in MDA-MB-231 cells following BANCR knockdown. The expression of (F) MMP2, (G) MMP9, (H) MMP14, (I) Vimentin and (J) E-cadherin was analyzed in MDA-MB-468 cells following BANCR overexpression. Statistical significance was determined using Student's t-test between indicated columns in (A-J). ${ }^{* *} \mathrm{P}<0.01$. NC, negative control; BNCR, BRAF-regulated lncRNA 1; MMP, matrix metallopeptidase; E-cadherin, epithelial cadherin.

Furthermore, high BANCR expression was indicated to be an independent risk factor for patients with $\mathrm{BC}$ with poor OS and RFS rates. In addition, the functional role of BANCR promoting $\mathrm{BC}$ metastasis and proliferation was demonstrated in BC cells. These observations indicated that BANCR may serve as a promising prognostic marker and therapeutic target in $\mathrm{BC}$.

\section{Acknowledgements}

Not applicable.

\section{Funding}

No funding was received. 


\section{Availability of data and materials}

Not applicable.

\section{Authors' contributions}

JJ collected the clinical specimens and conducted RT-qPCR, performed in vitro studies and wrote the manuscript. SHS, XJL and LS performed RT-qPCR and statistical analysis. QDG and CL collected the clinical specimens and wrote the manuscript. WZ designed the study.

\section{Ethics approval and consent to participate}

The present study was approved by the Ethics Committee of the Ningbo No. 2 Hospital.

\section{Consent for publication}

Written informed consent was obtained from each patient.

\section{Competing interests}

The authors declare that they have no competing interests.

\section{References}

1. Chen W, Zheng R, Baade PD, Zhang S, Zeng H, Bray F, Jemal A, Yu XQ and He J: Cancer statistics in China, 2015. CA Cancer J Clin 66: 115-132, 2016.

2. Cancer Genome Atlas Network: Comprehensive molecular portraits of human breast tumours. Nature 490: 61-70, 2012.

3. Ciriello G, Gatza ML, Beck AH, Wilkerson MD, Rhie SK, Pastore A, Zhang H, McLellan M, Yau C, Kandoth C, et al: Comprehensive molecular portraits of invasive lobular breast cancer. Cell 163: 506-519, 2015.

4. Boon RA, Jaé N, Holdt L and Dimmeler S: Long noncoding RNAs: From clinical genetics to therapeutic targets? J Am Coll Cardiol 67: 1214-1226, 2016.

5. Bhan A and Mandal SS: Long noncoding RNAs: Emerging stars in gene regulation, epigenetics and human disease. Chem Med Chem 9: 1932-1956, 2014.

6. Ponting CP, Oliver PL and Reik W: Evolution and functions of long noncoding RNAs. Cell 136: 629-641, 2009.

7. Ulitsky I and Bartel DP: lincRNAs: Genomics, evolution, and mechanisms. Cell 154: 26-46, 2013.

8. Isin M and Dalay N: LncRNAs and neoplasia. Clin Chim Acta 444: 280-288, 2015.

9. Li X, Wu Z, Fu X and Han W: lncRNAs: Insights into their function and mechanics in underlying disorders. Mutat Res Rev Mutat Res 762: 1-21, 2014.

10. Maass PG, Luft FC and Bähring S: Long non-coding RNA in health and disease. J Mol Med (Berl) 92: 337-346, 2014.

11. Huang NS, Chi YY, Xue JY, Liu MY, Huang S, Mo M, Zhou SL and $\mathrm{Wu} \mathrm{J}$ : Long non-coding RNA metastasis associated in lung adenocarcinoma transcript 1 (MALAT1) interacts with estrogen receptor and predicted poor survival in breast cancer. Oncotarget 7: 37957-37965, 2016

12. Si X, Zang R, Zhang E, Liu Y, Shi X, Zhang E, Shao L, Li A, Yang N, Han X, et al: LncRNA H19 confers chemoresistance in ERo-positive breast cancer through epigenetic silencing of the pro-apoptotic gene BIK. Oncotarget 7: 81452-81462, 2016.

13. Xu S, Sui S, Zhang J, Bai N, Shi Q, Zhang G, Gao S, You Z, Zhan C, Liu F and Pang D: Downregulation of long noncoding RNA MALAT1 induces epithelial-to-mesenchymal transition via the PI3K-AKT pathway in breast cancer. Int J Clin Exp Pathol 8: 4881-4891, 2015.

14. Milevskiy MJ, Al-Ejeh F, Saunus JM, Northwood KS, Bailey PJ, Betts JA, McCart Reed AE, Nephew KP, Stone A, Gee JM, et al: Long-range regulators of the lncRNA HOTAIR enhance its prognostic potential in breast cancer. Hum Mol Genet 25: 3269-3283, 2016.
15. Deng LL, Chi YY, Liu L, Huang NS, Wang L and Wu J: LINC00978 predicts poor prognosis in breast cancer patients. Sci Rep 6: 37936, 2016.

16. Xu SP, Zhang JF, Sui SY, Bai NX, Gao S, Zhang GW, Shi QY, You ZL, Zhan C and Pang D: Downregulation of the long noncoding RNA EGOT correlates with malignant status and poor prognosis in breast cancer. Tumor Biol 36: 9807-9812, 2015.

17. Mendell JT: Targeting a long noncoding RNA in breast cancer. N Engl J Med 374: 2287-2289, 2016.

18. Yang Y, Qian J, Xiang Y, Chen Y and Qu J: The prognostic value of long noncoding RNA HOTTIP on clinical outcomes in breast cancer. Oncotarget 8: 6833-6844, 2017.

19. Flockhart RJ, Webster DE, Qu K, Mascarenhas N, Kovalski J, Kretz M and Khavari PA: BRAFV600E remodels the melanocyte transcriptome and induces BANCR to regulate melanoma cell migration. Genome Res 22: 1006-1014, 2012

20. McCarthy N: Epigenetics. Going places with BANCR. Nat Rev Cancer 12: 451, 2012.

21. Liu Z, Yang T, Xu Z and Cao X: Upregulation of the long non-coding RNA BANCR correlates with tumor progression and poor prognosis in esophageal squamous cell carcinoma. Biomed Pharmacother 82: 406-412, 2016.

22. Su S, Gao J, Wang T, Wang J, Li H and Wang Z: Long non-coding RNA BANCR regulates growth and metastasis and is associated with poor prognosis in retinoblastoma. Tumor Biol 36: 7205-7211, 2015.

23. Jiang W, Zhang D, Xu B, Wu Z, Liu S, Zhang L, Tian Y, Han X and Tian D: Long non-coding RNA BANCR promotes proliferation and migration of lung carcinoma via MAPK pathways. Biomed Pharmacother 69: 90-95, 2015.

24. Peng ZQ, Lu RB, Xiao DM and Xiao ZM: Increased expression of the lncRNA BANCR and its prognostic significance in human osteosarcoma. Genet Mol Res 15, 2016.

25. Fan YH, Ye MH, Wu L, Wu MJ, Lu SG and Zhu XG: BRAF-activated lncRNA predicts gastrointestinal cancer patient prognosis: A meta-analysis. Oncotarget 8: 6295-6303, 2017.

26. Amin MB, Edge SB, Greene FL, Byrd DR, Brookland RK, Washington MK, Gershenwald JE, Compton CC, Hess KR, Sullivan DC, et al (eds); American Joint Committee on Cancer (AJCC): AJCC Cancer Staging Manual. 8th edition. Springer, New York, NY, 2017.

27. Livak KJ and Schmittgen TD: Analysis of relative gene expression data using real-time quantitative PCR and the 2(-Delta Delta C(T)) method. Methods 25: 402-408, 2001.

28. Tsai JH and Yang J: Epithelial-mesenchymal plasticity in carcinoma metastasis. Genes Dev 27: 2192-2206, 2013.

29. Tsai MC, Spitale RC and Chang HY: Long intergenic noncoding RNAs: New links in cancer progression. Cancer Res 71: 3-7, 2011.

30. Liu H, Li J, Koirala P, Ding X, Chen B, Wang Y, Wang Z, Wang C, Zhang $\mathrm{X}$ and Mo $\mathrm{YY}$ : Long non-coding RNAs as prognostic markers in human breast cancer. Oncotarget 7: 20584-20596, 2016.

31. Chen QN, Wei CC, Wang ZX and Sun M: Long non-coding RNAs in anti-cancer drug resistance. Oncotarget 8: 1925-1936, 2017.

32. Vennin C, Spruyt N, Robin YM, Chassat T, Le Bourhis X and Adriaenssens E: The long non-coding RNA 91H increases aggressive phenotype of breast cancer cells and up-regulates H19/IGF2 expression through epigenetic modifications. Cancer Lett 385: 198-206, 2017

33. Sas-Chen A, Aure MR, Leibovich L, Carvalho S, Enuka Y, Körner C, Polycarpou-Schwarz M, Lavi S, Nevo N, Kuznetsov Y, et al: LIMT is a novel metastasis inhibiting lncRNA suppressed by EGF and downregulated in aggressive breast cancer. EMBO Mol Med 8: 1052-1064, 2016.

34. Gökmen-Polar Y, Vladislav IT, Neelamraju Y, Janga SC and Badve S: Prognostic impact of HOTAIR expression is restricted to ER-negative breast cancers. Sci Rep 5: 8765, 2015.

35. Chen JX, Chen M, Zheng YD, Wang SY and Shen ZP: Up-regulation of BRAF activated non-coding RNA is associated with radiation therapy for lung cancer. Biomed Pharmacother 71: 79-83, 2015.

36. Zheng $\mathrm{H}$, Wang M, Jiang L, Chu H, Hu J, Ning J, Li B, Wang D and Xu J: BRAF-activated long noncoding RNA modulates papillary thyroid carcinoma cell proliferation through regulating thyroid stimulating hormone receptor. Cancer Res Treat 48: 698-707, 2016. 
37. Wang D, Wang D, Wang N, Long Z and Ren X: Long non-coding RNA BANCR promotes endometrial cancer cell proliferation and invasion by regulating MMP2 and MMP1 viaERK/MAPK signaling pathway. Cell Physiol Biochem 40: 644-656, 2016.

38. Li R, Zhang L, Jia L, Duan Y, Li Y, Bao L and Sha N: Long non-coding RNA BANCR promotes proliferation in malignant melanoma by regulating MAPK pathway activation. PLoS One 9: e100893, 2014

39. Zhang ZX, Liu ZQ, Jiang B, Lu XY, Ning XF, Yuan CT and Wang AL: BRAF activated non-coding RNA (BANCR) promoting gastric cancer cells proliferation via regulation of NF- $\kappa \mathrm{B} 1$. Biochem Biophys Res Commun 465: 225-231, 2015.

40. Li L, Zhang L, Zhang Y and Zhou F: Increased expression of LncRNA BANCR is associated with clinical progression and poor prognosis in gastric cancer. Biomed Pharmacother 72: 109-112, 2015.
41. Scaria V, Shi Y, Liu Y, Jie D, Yun T, Li W, Yan L, Wang K and Feng J: Downregulated long noncoding RNA BANCR promotes the proliferation of colorectal cancer cells via downregualtion of p21 expression. PloS One 10: e0122679, 2015.

42. Guo Q, Zhao Y, Chen J, Hu J, Wang S, Zhang D and Sun Y: BRAF-activated long non-coding RNA contributes to colorectal cancer migration by inducing epithelial-mesenchymal transition. Oncol Lett 8: 869-887, 2014.

43. Sun M, Liu XH, Wang KM, Nie FQ, Kong R, Yang JS, Xia R, Xu TP, Jin FY, Liu ZJ, et al: Downregulation of BRAF activated non-coding RNA is associated with poor prognosis for non-small cell lung cancer and promotes metastasis by affecting epithelial-mesenchymal transition. Mol Cancer 13: 68, 2014. 